\title{
Risk Factors for Post-ERCP Pancreatitis: A Prospective Multicenter Study
}

Chi-Liang Cheng, M.D., ${ }^{1}$ Stuart Sherman, M.D., ${ }^{1}$ James L. Watkins, M.D., ${ }^{2}$ Jeffrey Barnett, M.D., ${ }^{3}$ Martin Freeman, M.D., ${ }^{4}$ Joseph Geenen, M.D. ${ }^{5}$ Michael Ryan, M.D., ${ }^{6}$ Harrison Parker, M.D., ${ }^{7}$ James T. Frakes, M.D., ${ }^{8}$ Evan L. Fogel, M.D., ${ }^{1}$ William B. Silverman, M.D. ${ }^{9}$ Kulwinder S. Dua, M.D., ${ }^{10}$ Giuseppe Aliperti, M.D., ${ }^{11}$ Paul Yakshe, M.D., ${ }^{12}$ Michael Uzer, M.D., ${ }^{13}$ Whitney Jones, M.D., ${ }^{14}$ John Goff, M.D., ${ }^{15}$ Laura Lazzell-Pannell, RN, ${ }^{1}$ Abdullah Rashdan, M.D., ${ }^{1}$ M'hamed Temkit, MS, ${ }^{1}$ and Glen A. Lehman, M.D. ${ }^{1}$ ${ }^{1}$ Indiana University Medical Center, Indianapolis, Indiana; ${ }^{2}$ University of Wisconsin, Madison, Wisconsin;

${ }^{3}$ University of Michigan, Ann Arbor, Michigan; ${ }^{4}$ Hennepin County Medical Center, Minneapolis, Minnesota;

${ }^{5}$ GI Consultants Ltd., Milwaukee, Wisconsin; ${ }^{6}$ The Marshfield Clinic, Marshfield, Wisconsin; ${ }^{7}$ Milwaukee GI Specialists, Milwaukee, Wisconsin; ${ }^{8}$ Rockford Gastroenterology Associates, Ltd., Rockford, Illinois; ${ }^{9}$ University of Pittsburgh, Pittsburgh, Pennsylvania; ${ }^{10}$ Medical College of Wisconsin, Milwaukee, Wisconsin; ${ }^{11}$ Washington University, St. Louis, Missouri; ${ }^{12}$ University of Minnesota Hospital, Minneapolis, Minnesota; ${ }^{13}$ Rush Presbyterian, Chicago, Illinois; ${ }^{14}$ University of Louisville, Louisville, Kentucky; ${ }^{15}$ Gastroenterology Associates, P.C., Arvada, Colorado

OBJECTIVES: Pancreatitis is the most common and serious complication of diagnostic and therapeutic ERCP. The aim of this study is to examine the potential patient- and procedure-related risk factors for post-ERCP pancreatitis in a prospective multicenter study.

METHODS: A 160-variable database was prospectively collected by a defined protocol on patients undergoing diagnostic or therapeutic ERCP at 15 centers in the Midwest Pancreaticobiliary Group and participating in a randomized controlled study evaluating whether prophylactic corticosteroids will reduce the incidence of post-ERCP pancreatitis. Data were collected prior to the procedure, at the time of procedure, and 24-72 $\mathrm{h}$ after discharge. Post-ERCP pancreatitis was diagnosed and its severity graded according to consensus criteria.

RESULTS: $\quad$ Of the 1,115 patients enrolled, diagnostic ERCP with or without sphincter of Oddi manometry (SOM) was performed in $536(48.1 \%)$ and therapeutic ERCP in 579 (51.9\%). Suspected sphincter of Oddi dysfunction (SOD) was the indication for the ERCP in 378 patients (33.9\%). Pancreatitis developed in 168 patients $(15.1 \%)$ and was graded mild in $112(10 \%)$, moderate in $45(4 \%)$, and severe in 11(1\%). There was no difference in the incidence of pancreatitis or the frequency of investigated potential pancreatitis risk factors between the corticosteroid and placebo groups. By univariate analysis, the incidence of post-ERCP pancreatitis was significantly higher in 19 of 30 investigated variables. In the multivariate risk model, significant risk factors with adjusted odds ratios (OR) were: minor papilla sphincterotomy (OR: 3.8), suspected SOD (OR: 2.6), history of post-ERCP pancreatitis (OR: 2.0), age $<60 \mathrm{yr}$ (OR: 1.6), $\geq 2$ contrast injections into the pancreatic duct (OR: 1.5), and trainee involvement (OR: 1.5). Female gender, history of recurrent idiopathic pancreatitis, pancreas divisum, SOM, difficult cannulation, and major papilla sphincterotomy (either biliary or pancreatic) were not multivariate risk factors for post-ERCP pancreatitis.

CONCLUSION: This study emphasizes the role of patient factors (age, SOD, prior history of post-ERCP pancreatitis) and technical factors (number of PD injections, minor papilla sphincterotomy, and operator experience) as the determining high-risk predictors for post-ERCP pancreatitis.

(Am J Gastroenterol 2006;101:139-147)

\section{INTRODUCTION}

Pancreatitis is the most common and feared complication of ERCP, occurring in up to $30-40 \%$ of high-risk patients $(1-3)$. It is associated with substantial morbidity and occasional mortality. There have been ongoing efforts to minimize the incidence and severity of post-ERCP pancreatitis. Precise identification of risk factors for post-ERCP pancreatitis is essential to recognize high-risk cases in which ERCP should be avoided if possible, or in which protective endoscopic or pharmacologic interventions should be considered $(2,4)$. The mechanisms that lead to post-ERCP pancreatitis are not 
fully understood but believed to be multifactorial (mechanical, chemical, hydrostatic, enzymatic, microbiologic, and thermal), and the triggering event is thought to cause premature intracellular activation of proteolytic enzymes within acinar cells resulting in cellular injury and autodigestion of pancreatic tissue $(1,5)$.

A number of specific risk factors, acting independently or in concert, have been proposed as predictors of post-ERCP pancreatitis. These include both patient- and procedurerelated factors. However, the reported risk factors vary widely from study to study. These discrepancies may be attributable to heterogeneous patient populations, differing levels of endoscopic expertise, varying cannulation techniques, and disparate definition of post-ERCP pancreatitis. More important, the use of univariate analysis to identify risk factors for postERCP pancreatitis may produce misleading results because of inability to sort out confounding variables (6-10). Recent studies have used multivariate analysis as a tool to identify and quantify the effect of multiple potentially confounding risk factors (11-20).

The aim of this study was to use multivariate analysis and examine the independent risk factors for post-ERCP pancreatitis in a group of patients who were prospectively randomized to receive prophylactic corticosteroids or placebo in a double-blinded, multicenter, controlled clinical trial.

\section{MATERIALS AND METHODS}

Physicians from 15 participating endoscopic centers affiliated with the Midwest Pancreaticobiliary Group conducted a randomized, prospective, placebo-controlled study to evaluate whether prophylactic administration of corticosteroids reduces the risk and severity of post-ERCP pancreatitis. Data were prospectively collected to determine the patient- and procedure-related risk factors for post-ERCP pancreatitis. The research proposal was approved by the institutional review boards at all participating centers.

\section{Patients}

Patients were recruited serially as they presented for diagnostic or therapeutic ERCP. Patients were excluded from the study for any of the following reasons: (1) age less than $18 \mathrm{yr}$, (2) pregnancy, (3) mental disability, (4) incarceration, (5) active pancreatitis before procedure, (6) allergy to corticosteroids, (7) actual treatment with corticosteroids, (8) active tuberculosis, (9) uncontrolled diabetes, (10) contrast allergy, (11) known hepatitis B, (12) active bacteremia or septicemia, (13) planned biliary stent removal or exchange without planned pancreatogram, or (14) need for emergent ERCP within $12 \mathrm{~h}$.

\section{Study Protocol}

After obtaining informed consent, enrolled patients were randomized to receive either prednisone, $40 \mathrm{mg}$ by mouth, 15 $\mathrm{h}( \pm 3 \mathrm{~h})$ and $3 \mathrm{~h}( \pm 1 \mathrm{~h})$ before ERCP, or a placebo (similar pill without the active drug) given at similar time intervals. Randomization was accomplished in a blinded fashion by pharmacy staff. In sequence, concealed envelops with active drug or placebo were dispensed. Baseline values for biochemical tests of liver function, amylase and lipase, and a complete blood count (CBC) were determined before the procedure. ERCP was performed by using the standard high-osmolality ionic-contrast agent diatrizoate meglumine (Hypaque, Winthrop Pharmaceuticals, New York, NY). A $\mathrm{CBC}$ and serum amylase and lipase levels were obtained at 2-4 h after the ERCP was completed. Patients were prospectively evaluated for the development of post-ERCP pancreatitis. Admission of an outpatient or prolongation of current hospitalization was at the discretion of the managing physician. Patients discharged on the day the ERCP was performed were contacted by telephone during the 24$72 \mathrm{~h}$ following the procedure to assess their clinical condition and evaluate for the development of postprocedure pancreatitis.

\section{Definitions}

Suspected sphincter of Oddi dysfunction (SOD) referred to the pre-ERCP suspicion of a functional or structural abnormality of the sphincter of Oddi, independent of any manometric findings, thought to be the cause of recurrent abdominal pain or pancreatitis. Cannulation difficulty was determined on the basis of the number of attempts on the papilla with a cannulation instrument (both common bile duct and pancreatic duct). Cannulation was judged easy when one to eight attempts took place and moderate/difficult when more than eight occurred. The number of pancreatic duct injections was the total number of times any volume of contrast was injected into the pancreatic duct. Acinarization was defined as any fluoroscopically observed focal or diffuse pancreatic parenchymal blush of contrast. Bile duct diameter was the measured maximal duct diameter within $2 \mathrm{~cm}$ of the papilla adjusted for radiographic magnification. Chronic pancreatitis was defined as the presence of mild, moderate, or severe pancreatographic abnormalities according to Cambridge criteria (21).

The definition of pancreatitis and the grading of its severity were based on consensus criteria (3). Post-ERCP pancreatitis was diagnosed when new-onset or increased abdominal pain lasted for more than $24 \mathrm{~h}$, caused an unplanned admission of an outpatient for more than one night or prolonged a planned hospitalization of an inpatient, and was associated with an increase in serum amylase level of at least three times greater than the normal upper limit at approximately $18 \mathrm{~h}$ (the next morning) after the procedure. The severity of the pancreatitis was graded mild when hospitalization lasted 2-3 days, moderate when 4-10 days, and severe when hospitalization was prolonged for more than 10 days or any of the following occurred: hemorrhagic pancreatitis, pancreatic necrosis, pancreatic pseudocyst, or a need for percutaneous drainage or surgery. 


\section{Data Management}

Demographic, procedure, and follow-up data were prospectively collected at the time of the procedure, before, and 24-72 $\mathrm{h}$ after discharge, and entered into a 160-variable database. The database was developed using specific software (Foxpro, Microsoft Corp., Seattle, WA) with screen entry and built-in edit checks to maintain the quality of the data. The clinical centers were asked to send copies of the data form to the coordinating center. The data were then entered by a nurse coordinator and checked by a second coordinator.

\section{Statistical Analysis}

The primary outcome analyzed was development of postERCP pancreatitis. Risk factors for pancreatitis were assessed using univariate analysis with $\chi^{2}$ test for categorical variables and simple logistic regression for continuous variables. Variables with a $p$ value less than 0.2 in the univariate analysis were all included in a forward stepwise multiple logistic regression model to identify the independent risk factors for pancreatitis. Goodness-of-fit for the final multivariate model was assessed by the 2-log likelihood criterion. Statistical analyses were performed using the statistical software package SAS version 8.2 (SAS Institute, Cary, NC).

\section{RESULTS}

A total of 1,115 patients (396 men, 719 women; mean age 51.2 [16.7] yr) were enrolled into the study (Table 1). Five hundred fifty-five patients were randomized to the prednisone group and 560 to the placebo group. The rates of post-ERCP pancreatitis in those patients receiving oral prednisone and those receiving placebo have been reported previously (22). Because the frequency of post-ERCP pancreatitis $(16.6 \%$ receiving prednisone $v s 13.6 \%$ in the placebo group, $p=0.19$ ) and the risk factors for pancreatitis were similar for both groups, the data presented here will be combined treatment and placebo groups unless otherwise stated.

Three hundred and seventy-eight patients (33.9\%) were evaluated for suspected SOD. A prior history of postERCP pancreatitis was noted in 81 patients (7.3\%). Diagnostic ERCP with or without sphincter of Oddi manometry (SOM) was performed in 536 patients $(48.1 \%)$, while 579 patients $(51.9 \%)$ underwent therapeutic procedures. Endoscopic sphincterotomies were performed in 577 patients

Table 1. Patient Characteristics

\begin{tabular}{lc}
\hline Number of patients & 1115 \\
\hline \% female & $64.5 \%$ \\
Mean age & 51.2 \\
Number older than $60 \mathrm{yr}$ old & $362(32.5 \%)$ \\
Mean BMI & $26.8 \mathrm{~kg} / \mathrm{m}^{2}$ \\
Prior pancreatitis & $433(38.8 \%)$ \\
Suspected SOD & $378(33.9 \%)$ \\
Chronic pancreatitis & $192(16.1 \%)$ \\
\hline
\end{tabular}

BMI = body mass index; SOD = sphincter of Oddi dysfunction.
$(51.7 \%)$; sphincterotomies were biliary alone in 381 patients (34.2\%), pancreatic alone (major and/or minor papilla) in 126 patients $(11.3 \%)$, and dual pancreaticobiliary in 70 patients (6.3\%). Manometry was performed in 395 patients $(35.4 \%)$; SOM was biliary alone in 98 patients $(8.8 \%)$, pancreatic alone in 47 (4.2\%), and both biliary and pancreatic in $250(22.4 \%)$. Precut sphincterotomy was performed in 103 patients $(9.2 \%)$. Pancreas divisum was found in 123 patients $(11.0 \%)$ and minor papilla sphincterotomy was performed in $50(4.5 \%)$. Chronic pancreatitis was found in 192 patients $(17.2 \%)$.

Post-ERCP pancreatitis occurred in 168 patients $(15.1 \%)$. It was graded as mild in 112 patients $(10 \%)$, moderate in 45 (4\%), and severe in $11(1 \%)$. Pancreatitis occurred after 63 $(11.8 \%)$ of 536 ERCPs that were diagnostic alone and 105 (18.1\%) of 579 procedures that were therapeutic.

\section{Univariate Analysis}

In the univariate analysis, 11 of 15 patient-related factors and 8 of 15 procedure-related factors were found to be statistically significantly associated with an increased risk of post-ERCP pancreatitis (Table 2). Technical factors that significantly influenced the risk of pancreatitis included: minor papilla sphincterotomy, major papilla pancreatic sphincterotomy, moderate/difficult cannulation, performance of SOM, pancreatic stent placement, minor papilla cannulation, and therapeutic ERCP procedure. Trainee involvement and $\geq 2$ pancreatic duct injection with contrast were almost significant in the univariate analysis. Acinarization, precut sphincterotomy, biliary sphincterotomy, biliary orifice balloon dilation, and prior failed ERCP were not significant risk factors.

Significant patient-related risk factors by univariate analysis included: history of post-ERCP pancreatitis, pancreas divisum, suspected SOD, recurrent idiopathic pancreatitis, prior cholecystectomy, recurrent abdominal pain, age $<60$ yr, female sex, periampullary diverticulum, presence of common bile duct stone, and obstructive jaundice. Body mass index, distal bile duct diameter, absence of chronic pancreatitis, and prednisone premedication were not significant risk factors.

\section{Multivariate Analysis}

Only those risk factors that reached a $p$ value cutoff 0.20 or less were used to create the multivariate model. Table 3 shows the results of the stepwise multiple logistic regression from the pool of 21 potential risk factors in the univariate analysis. Six risk factors were significant by multivariate analysis, three were characteristics of the patients (suspected SOD, younger age, prior history of post-ERCP pancreatitis), and three were procedure-related ( $\geq 2$ pancreatic duct injections, minor papilla sphincterotomy, trainee involvement). There was no evidence of multicollinearity issues or lack of fit. The Hosmer-Lemeshow goodness of fit test was not significant at the 0.05 significance level and the C-statistic was 0.692 (which nears 0.70 , the value of acceptable predictive power).

Of the 11 cases with severe post-ERCP pancreatitis, the risk factors are shown in Table 4. One patient had four 
Table 2. Univariate Analysis of Risk Factors for Post-ERCP Pancreatitis

\begin{tabular}{|c|c|c|c|}
\hline \multirow[b]{2}{*}{ Variables } & \multicolumn{3}{|c|}{ Post-ERCP Pancreatitis Incidence (\%) } \\
\hline & With Variable & Without Variable & $p$ Value \\
\hline \multicolumn{4}{|l|}{ Patient-related variables } \\
\hline \multicolumn{4}{|l|}{ Significant } \\
\hline History of previous post-ERCP pancreatitis & 23/81 (28.4) & 145/1034 (14.0) & 0.0005 \\
\hline Pancreas divisum & $30 / 123(24.4)$ & 138/992 (13.9) & 0.002 \\
\hline Suspected sphincter of Oddi dysfunction & $92 / 378(24.3)$ & 76/737 (10.3) & $<0.0001$ \\
\hline Recurrent idiopathic pancreatitis & $46 / 221(20.8)$ & $122 / 894(13.6)$ & 0.008 \\
\hline Prior cholecystectomy & $64 / 327(19.6)$ & $104 / 788(13.2)$ & 0.007 \\
\hline Recurrent abdominal pain & $120 / 656(18.3)$ & $48 / 459(10.5)$ & 0.0003 \\
\hline Age $<60$ yr & $134 / 753(17.8)$ & $34 / 362(9.4)$ & 0.0002 \\
\hline Female sex & $120 / 719(16.7)$ & 48/396 (12.0) & 0.042 \\
\hline Periampullary diverticulum & $10 / 119(8.4)$ & 158/994 (15.9) & 0.03 \\
\hline Common bile duct stones & $18 / 232(7.8)$ & $150 / 883(17.0)$ & 0.0005 \\
\hline Obstructive jaundice & $10 / 145(6.9)$ & $158 / 970(16.3)$ & 0.003 \\
\hline \multicolumn{4}{|l|}{ Not significant } \\
\hline Body mass index $<30 \mathrm{~kg} / \mathrm{m}^{2}$ & $133 / 702(18.9)$ & $54 / 413(13.1)$ & 0.10 \\
\hline Prednisone premedication & $92 / 555(16.6)$ & $76 / 560(13.6)$ & 0.19 \\
\hline Distal bile duct diameter $<5 \mathrm{~mm}$ & 47/302 (15.6) & $99 / 688(14.4)$ & 0.63 \\
\hline Absence of chronic pancreatitis & $137 / 923(14.8)$ & $31 / 192(16.1)$ & 0.28 \\
\hline \multicolumn{4}{|l|}{ Procedure-related variables } \\
\hline \multicolumn{4}{|l|}{ Significant } \\
\hline Minor papilla sphincterotomy & $17 / 50(34.0)$ & $151 / 1065(14.2)$ & 0.0001 \\
\hline Major papilla pancreatic sphincterotomy & $30 / 146(20.6)$ & $138 / 969(14.2)$ & 0.0485 \\
\hline Pancreatic sphincterotomy (major + minor) & $47 / 196(24.0)$ & $121 / 919(13.2)$ & 0.002 \\
\hline Moderate/difficult cannulation & $36 / 137(26.3)$ & $132 / 978(13.5)$ & $<0.0001$ \\
\hline Sphincter of Oddi manometry & $94 / 395(23.8)$ & $74 / 720(10.3)$ & $<0.0001$ \\
\hline Pancreatic stent placement & $49 / 218(22.5)$ & $119 / 897(13.3)$ & 0.0007 \\
\hline Minor papilla cannulation & $31 / 146(21.2)$ & $137 / 969(14.1)$ & 0.03 \\
\hline Therapeutic ERCP procedure & $105 / 579(18.1)$ & $63 / 536(11.8)$ & 0.003 \\
\hline \multicolumn{4}{|l|}{ Not significant } \\
\hline Acinarization & $11 / 59(18.6)$ & $157 / 1056(14.9)$ & 0.43 \\
\hline Precut sphincterotomy & 19/103 (18.4) & $149 / 1012(14.7)$ & 0.31 \\
\hline Biliary sphincterotomy & $74 / 451(16.4)$ & 94/664 (14.1) & 0.30 \\
\hline Trainee involvement & $100 / 588(17.0)$ & 68/524 (13.0) & 0.06 \\
\hline$\geq 2$ pancreatic duct injections & $103 / 613(16.8)$ & $65 / 502(13.0)$ & 0.07 \\
\hline Prior failed ERCP & $22 / 156(14.1)$ & $146 / 959(15.2)$ & 0.72 \\
\hline Biliary orifice balloon dilatation & $3 / 23(13.0)$ & $165 / 1092(15.1)$ & 0.78 \\
\hline
\end{tabular}

independent risk factors for pancreatitis, four patients had three, five patients had two, and one patient had one. Four of these patients had successful pancreatic stent placement. The average days of hospitalization were 18 days and one patient died from the post-ERCP pancreatitis.

Table 3. Significant Risk Factors for Post-ERCP Pancreatitis by Multivariate Analysis

\begin{tabular}{lccc}
\hline $\begin{array}{l}\text { Risk } \\
\text { Factors }\end{array}$ & $\begin{array}{c}\text { Odds } \\
\text { Ratio }\end{array}$ & $\begin{array}{c}95 \% \text { Confidence } \\
\text { Interval }\end{array}$ & $p$ Value \\
\hline $\begin{array}{l}\text { Minor papilla } \\
\text { sphincterotomy }\end{array}$ & 3.8 & $2.003-7.106$ & $<0.0001$ \\
$\begin{array}{c}\text { Suspected sphincter of } \\
\quad \text { Oddi dysfunction }\end{array}$ & 2.6 & $1.828-3.717$ & $<0.0001$ \\
$\begin{array}{c}\text { History of post-ERCP } \\
\text { pancreatitis }\end{array}$ & 2.0 & $1.186-3.448$ & 0.01 \\
$\begin{array}{l}\text { Age }<60 \text { yr } \\
\geq 2 \text { pancreatic } \\
\quad \text { duct injections }\end{array}$ & 1.6 & $1.033-2.402$ & 0.04 \\
\begin{tabular}{l} 
Trainee involvement \\
\hline
\end{tabular} & 1.5 & $1.046-2.103$ & 0.03 \\
\hline
\end{tabular}

\section{DISCUSSION}

The primary aim of this multicenter, randomized controlled study was to evaluate the effect of corticosteroid on the incidence of post-ERCP pancreatitis. Unfortunately, this study and others found that prednisone did not reduce the incidence or severity of post-ERCP pancreatitis (22). Identification of patient- and procedure-related risk factors for post-ERCP pancreatitis was prospectively evaluated as a secondary goal of this study. This study suggests that the risk of pancreatitis

Table 4. Risk Factors of the 11 Cases with Severe Post-ERCP Pancreatitis

\begin{tabular}{lc}
\hline Risk Factors & No. (Percentage) \\
\hline Age $<60$ yr & $8(73 \%)$ \\
Trainee involvement & $8(73 \%)$ \\
Suspected sphincter of Oddi dysfunction & $7(63 \%)$ \\
$\geq 2$ pancreatic duct injections & $7(63 \%)$ \\
Minor papilla sphincterotomy & $1(9 \%)$ \\
History of post-ERCP pancreatitis & $1(9 \%)$ \\
\hline
\end{tabular}


is determined as much by patient characteristics as by endoscopic techniques or maneuvers. The overall post-ERCP pancreatitis rate is $15.1 \%$, which is higher than previous reports and perhaps can be explained by a higher proportion of patients with suspected SOD (33.9\%) in our study population. In previous prospective large-scale risk factor studies, the reported pancreatitis rates ranged from $1.3 \%$ to $8.6 \%$, and the proportion of SOD patients was usually less than $10 \%(11-18)$.

The results of our study are in agreement with prior prospective multivariate analysis studies with regard to suspected $\operatorname{SOD}(11,12,19)$, younger age $(11,16,18,19)$, history of post-ERCP pancreatitis $(12-14)$, and pancreatic duct contrast injections $(11,12,15,18,19)$ as independent predictors for post-ERCP pancreatitis. Minor papilla sphincterotomy was shown in the current study to be a significant risk factor by univariate and multivariate analysis, which was not evaluated in the previous reports. Freeman et al. evaluated pancreatic sphincterotomy and considered it as an independent risk factor for pancreatitis, but they did not separate out minor and major pancreatic sphincterotomy in that study (12). There are conflicting data for the effect of endoscopic experience as a significant risk factor for post-ERCP pancreatitis $(11,12,17)$. Our results showed that trainee participation was a significant risk factor by multivariate analysis.

One recent meta-analysis assessing risk factors for postERCP pancreatitis revealed that suspected SOD, history of post-ERCP pancreatitis, female sex, precut sphincterotomy, and pancreatic contrast injections were independent predictors for this complication (23). In a review that selectively included full-length English language reports with multivariable analysis of the relationship between patient, procedure, or operator factors and post-ERCP pancreatitis found younger age, SOD, multiple pancreatic duct contrast injections, difficulty in cannulation, and precut sphincterotomy as significant risk factors in more than one study (24). The following factors were analyzed but were not found to be significant for pancreatitis in any study: cholangitis, coagulopathy, previous gastrectomy, history of jaundice, emergency procedure, intramural injection, SOM, biliary drainage, length of sphincterotomy, and bleeding during sphincterotomy.

The multivariate method has become the desired form of data analysis, particularly for quantifying the independent risk of an individual variable on the outcome. However, the multivariate method is limited by the structure of the mathematical model and the coding of variables (25). Moreover, adequate number of outcome events is needed to ensure accurate $p$ values and risk estimate. Therefore, some variables may have minimal impact on the outcome despite achieving statistical significance, whereas other variables that fail to achieve the threshold of $p<0.05$ may still have substantial effect on the outcome (25). Because of the complex nature of post-ERCP pancreatitis, there are numerous variables to adjust for, many of which are closely interrelated and often simultaneously present in the same case; it may be difficult to precisely define risk factors with high degree of certainty.
For example, a young female with suspected SOD, who undergoes ERCP and SOM with or without endoscopic sphincterotomy that could involve a difficult cannulation along with multiple pancreatic duct contrast injections is at high-risk for post-ERCP pancreatitis. But what is the relative contribution of each of these factors to pancreatitis may not be clear yet since this scenario is very common among high-risk cases for post-ERCP pancreatitis. It is also likely that other unknown factors, for instance, at the cellular level (e.g., genetic susceptibility, hormonal or inflammatory mediators) may have a significant role in such individuals indicating further consideration.

Patients with suspected SOD carry a substantial risk for pancreatitis after any type of ERCP, whether diagnostic, manometric, or therapeutic (26). Our data show that a suspicion of SOD independently, nearly tripled the risk of postERCP pancreatitis to a frequency (24\%) that is comparable with that found in other studies $(9,11,12,23)$. Contrary to the widely held opinion that SOM is the cause for such a high risk $(27,28)$, recent multivariate analyses show that empirical biliary sphincterotomy or even diagnostic ERCP in suspected SOD cases has similarly high risk, so that the manometry appears to have been erroneously blamed by association rather than being the cause $(11,12)$. Furthermore, the widespread use of aspiration catheters for pancreatic duct manometry is likely to have nearly eliminated any additional risk of manometry from other ERCP instrumentation (29).

Placement of a prophylactic pancreatic stent in SOD patients has been shown to reduce the risk of post-ERCP pancreatitis $(30,31)$. The reason for the high susceptibility to post-ERCP pancreatitis among patients with suspected SOD remains unclear.

Mehta et al. conducted a study evaluating predictors of post-ERCP complications in patients with suspected choledocholithiasis and found that absence of common bile duct stones was an independent risk factor for post-ERCP pancreatitis (19). In this study, a younger patient (age $<59$ yr) undergoing biliary sphincterotomy for suspected choledocholithiasis without a stone in the bile duct had a $27 \%$ risk of developing post-ERCP pancreatitis. Although the diagnosis of SOD was not considered in this study, the results clearly showed the danger of performing ERCP to look for bile duct stones in patients with recurrent postcholecystectomy pain (in the absence of objective evidence for stones) because there is generally a low probability of finding stones in such patients and a high risk of causing pancreatitis. Magnetic resonance cholangiopancreatography and endoscopic ultrasound can provide useful information in many of these cases and are becoming widely available. These techniques may be preferable to ERCP for patients with equivocal evidence of biliary obstruction, especially those at high risk for post-ERCP pancreatitis (12).

A history of previous post-ERCP pancreatitis was another clinical risk factor for post-ERCP pancreatitis, and our findings are consistent with several recent multivariate risk factor studies (12-14). Our analysis showed that the risk in such 
patients was increased twofold (OR: 2.0). In the study by Freeman et al., a history of previous post-ERCP pancreatitis was found to be the most potent risk factor (OR: 5.4) (12). Like other reports, a history of previous noniatrogenic pancreatitis is only a risk factor by univariate analysis, but not by multivariate analysis $(11,12,14)$.

Younger age was significant risk factor for pancreatitis in our study and four other reports using multivariable analysis $(11,16,18,19)$. However, a different age cutoff was used in these studies with $60 \mathrm{yr}$ in 3 and $70 \mathrm{yr}$ in two studies, respectively. The progressive decline in pancreatic exocrine function with aging may protect older patients from pancreatic injury (32). Similarly, chronic pancreatitis was found to provide some protection against ERCP-induced pancreatitis, perhaps explained by atrophy and decreased enzymatic activity (12). However, absence of chronic pancreatitis was not a significant factor in the present study.

The finding of pancreatic contrast injections as an independent risk factor for post-ERCP pancreatitis is consistent with previous reports $(11,12,14,15,18,19)$. However, somewhat different from other studies, our results showed that pancreatic contrast injections was barely significant (OR: 1.5), and a difficult cannulation was not significant. A possible explanation for these findings is that, unlike other similar studies, prophylactic pancreatic stents were frequently used in our patient group (about $20 \%$ of all ERCP) to reduce post-ERCP pancreatitis. There have been several prospective studies showing that successful pancreatic stent placement might mitigate the risk of post-ERCP pancreatitis $(30,31,33)$. The higher postERCP pancreatitis rates in this series (by univariate analysis) in patients undergoing prophylactic pancreatic duct stenting is almost certainly related to the fact that the stents were placed in high-risk cases. Thus, the frequent use of prophylactic pancreatic stents may somewhat modify the apparent risk factors found in this study compared to traditional studies.

The mechanism of how contrast injection induces pancreatic ductal and acinar cell injury is not clear. Current evidence does not support the notion that nonionic contrast is more effective than conventional ionic contrast in preventing post-ERCP pancreatitis (1). The widely held concept is that hydrostatic injury from overfilling of the pancreatic duct with contrast material is a major cause of pancreatitis. Previous studies have demonstrated a ducto-interstitial-venous pathway, and if enough radiographic contrast is injected into the pancreatic duct, the collecting system of the kidney can be seen during ERCP (34). Roszler et al. reported an inadvertent urogram during ERCP was followed by pancreatitis in $45 \%$ of patients (35). The pathophysiologic basis for these observations is related to the occurrence of ductal epithelial or acinar injury, with resultant backflow of intraductal contents into the interstitial space and venous circulation. However, acinarization of the pancreas is probably less important than has been previously believed. Most studies showed acinarization of the pancreas was significant by univariate analysis only $(11,16,18)$. Like other recent studies, acinarization was not a significant risk factor by univariate analysis in our series $(12,14)$.

Difficult cannulation has been shown to be an independent risk factor for pancreatitis and may occur without any apparent pancreatic duct instrumentation (11-14). This suggests that trauma to the papilla and pancreatic sphincter with impaired pancreatic drainage may be important in the pathogenesis of pancreatitis. Although there is an association with the number of times the papilla was manipulated, the cutoff numbers vary widely, with six attempts in two studies $(11,12)$ and 20 attempts in one study (13). In the current study, however, moderate to difficult cannulation ( $>8$ attempts) was only significant in the univariate analysis and this was possibly due to frequent placement of pancreatic stents mitigating this risk factor as described above. The difficulty of cannulation is not easily quantifiable and interactions with time for cannulation, method of cannulating, and number of pancreatic duct injections may occur. Balloon dilation of the biliary sphincter to extract bile duct stone was an independent factor for pancreatitis in a large multicenter study with a fourfold increase in risk (12). Likewise, trauma to the pancreatic sphincter is suspected to be the cause of pancreatitis. In our report, however, biliary orifice balloon dilatation was not found to be a risk factor for post-ERCP pancreatitis. This may be due to the infrequent performance of biliary orifice dilation in this study.

Thermal injury of the papilla is exclusive to endoscopic sphincterotomy. Edema produced by electrocautery is a wellrecognized consequence (36). It is hypothesized that swelling around the pancreatic orifice may hinder the flow of pancreatic juice. Overall, performance of a standard biliary sphincterotomy does not appear to add significant independent risk of pancreatitis $(12,17,20)$. On the contrary, pancreatic sphincterotomy was found to be a significant risk of pancreatitis in a large multivariate analysis (12). Pancreatic duct stenting appears to be beneficial in reducing the incidence of pancreatitis after pancreatic sphincterotomy. Patel et al. conducted a prospective, randomized, controlled study, and the preliminary results showed that pancreatic duct stenting after pancreatic sphincterotomy was associated with a lower rate of pancreatitis but had not reached statistical significance (37). Coagulation current causes more tissue injury and edema than cutting current, and the use of pure cut current has been shown to cause less pancreatitis than with blended current sphincterotomy (38). In our series, we evaluated all types of endoscopic sphincterotomy and found only minor papilla sphincterotomy was an independent risk factor for post-ERCP pancreatitis (OR: 3.8). Biliary sphincterotomy was not a significant risk factor by univariate analysis. Pancreatic sphincterotomy of major papilla was significant only by univariate but not by multivariate analysis. According to our findings, pancreas divisum is not a risk factor unless minor papilla therapy is performed.

Precut papillotomy to gain access to the common bile duct was not a risk factor in this study. However, like another similar report (12), most of these procedures were performed by 
a few highly experienced endoscopists and often followed or preceded by placement of a pancreatic stent, which has been shown to reduce the risk of precutting (39). Increasing numbers of reports from tertiary referral centers also confirmed that complication rates were not different from standard sphincterotomy (40). However, precutting was associated with a higher risk of pancreatitis in two other multicenter studies involving endoscopists with varied experience in $\operatorname{ERCP}(11,16)$, and in the meta-analysis study (23). These observations suggest that the risk of precut papillotomy is highly operator dependent. Our data support the concept that early use of precut by expert endoscopists might be preferable to prolonged attempts at cannulation in patients at high risk for pancreatitis $(12,17)$.

Trainee participation has been previously evaluated in one large multicenter study and the result showed it was not a significant risk factor (12). In another report by Vandervoort et $a l$., the complications of ERCP performed solely by attending physician were not significantly different from those performed by fellows under the supervision of attending endoscopists, but detailed statistical data were not provided (13). In our series, trainee participation did increase the risk by multivariate analysis (OR: 1.5). The reason for this disagreement was not clear. A similar discrepancy was also noted in the evaluation of endoscopists' case volume as a risk factor for post-ERCP pancreatitis. Some series reported low case volume was not significant in the multivariate analysis (12, 15), while others considered it as independent risk factor of pancreatitis $(17,18)$. Low case volume was also an independent risk factor for postsphincterotomy bleeding in two large multicenter series $(11,12)$. In the prospective multicenter study by Freeman et al., sphincterotomy-induced complications were noted more frequently at university-affiliated referral centers than private practices, which the authors attributed to different case mix (11). All of these observations suggest that case mix is at least as important as expertise in determining risk of post-ERCP pancreatitis.

There is an escalating risk of post-ERCP pancreatitis in patients with multiple risk factors $(12,14)$. In the report by Freeman et al., a woman with normal serum bilirubin, easy cannulation, and a bile duct stone had about a 5\% chance of post-ERCP pancreatitis. If an even moderately difficult cannulation was encountered, the risk rose to more than $16 \%$. If such a patient also had suspected SOD and had a difficult cannulation, the risk of pancreatitis rose to more than $40 \%$ (12). Friedland et al. designed a bedside scoring system for the risk of post-ERCP pancreatitis based on the results of multivariate analysis (14). Independent risk factors included in the scoring system were new pain during ERCP, pancreatic ductal contrast injection, a prior history of post-ERCP pancreatitis, and difficult cannulation. The results showed that cases scoring 4 or fewer points (the low-risk group) had a $2 \%$ risk of post-ERCP pancreatitis, cases scoring between 5 and 8 points (the medium-risk group) had a $7 \%$ risk, and cases scoring more than 8 (the high-risk group) had a $28 \%$ risk.
Pharmacological methods to prevent post-ERCP pancreatitis have been studied for many years. Most drugs appear promising in initial randomized single-center trials; however, conflicting results are often obtained from larger, multicenter studies. Octreotide, steroids, and interleukin-10 are typical examples. From the meta-analysis report by Andriulli et al., somatostatin (an antisecretory agent) and gabexate mesilate (a protease inhibitor) are two effective prophylactic drugs for preventing post-ERCP pancreatitis (41). Neither drug is available in the United States. The number of patients that need to be treated with both drugs to prevent a single case of pancreatitis is 13-27. One recent report showed that a single dose of diclofenac (a nonsteroidal antiinflammatory agent, NSAID) suppository helped to reduce the incidence of post-ERCP pancreatitis (42). NSAIDs can inhibit the early inflammatory cascade involving phospholipase-A2, prostaglandins, or endothelial neutrophil attachment during acute pancreatitis. It should be appreciated that this is only a single-center study and diclofenac was not effective in the subgroup of patients with SOD, the very group of patients that are at greatest risk. A larger multicenter study is needed to confirm the protective role of NSAIDs.

In conclusion, multivariate analysis indicates that patientrelated risk factors are as important as technical ones. Until effective, safe, and low-cost prophylactic drugs are definitively identified and made available, selective use in high-risk groups may be warranted. Unfortunately, such a drug is not available in the United States. Risk stratification will allow endoscopists to better identify patients who are at significant risk and permit detailed informed consent as well as possibly defer examinations in the highest-risk groups. Appropriate technical "prophylaxis" during ERCP, such as pancreatic ductal stenting, should also be carefully considered in high-risk patients to prevent the development of pancreatitis.

\section{Appendix}

Other contributing authors: Klaus Gottlieb, MD, Indiana University Medical Center, Indianapolis, Indiana; Kevin Block, MD, Mark Reichelderfer, MD, University of Wisconsin, Madison, Wisconsin; Grace Elta, MD, William D. Chey, MD, University of Michigan, Ann Arbor, Michigan; Oliver Cass, MD, Hennepin County Medical Center, Minneapolis, Minnesota; Marc Catalano, MD, Michael Schmalz, MD, Daniel Geenen, MD, GI Consultants Ltd., Milwaukee, Wisconsin; Christopher Rall, MD, Marshfield Clinic, Marshfield, Wisconsin; David Carron, MD, Milwaukee GI Specialists, Milwaukee, Wisconsin; John Johanson, MD, Roger Greenlaw, MD, Mark Shiels, MD, Rockford Gastroenterology Associates, Ltd., Rockford, Illinois; Adam Slivka, MD, University of Pittsburgh, Pittsburgh, Pennsylvania; Anthony Bohorfoush, MD, Reza Shaker, MD, Medical College of Wisconsin, Milwaukee, Wisconsin; Hitesh Chokshi, MD, David Walden, MD, Washington University, St. Louis, Missouri; Raja Kaikaus, MD, University of Louisville, Louisville, Kentucky. 


\section{ACKNOWLEDGMENT}

This study was supported by AGA Foundation Smith Kline Beecham Clinical Research Award.

Reprint requests and correspondence: Stuart Sherman, M.D., Professor of Medicine and Radiology, Indiana University Medical Center, 550 N University Blvd, Suite 4100, Indianapolis, IN 46202. Received August 11, 2004; accepted August 9, 2005.

\section{REFERENCES}

1. Gottlieb K, Sherman S. ERCP and endoscopic biliary sphincterotomy-induced pancreatitis. Gastrointest Endosc Clin N Am 1998;8:87-114.

2. Freeman ML. Adverse outcomes of ERCP. Gastrointest Endosc 2002;56(Suppl):S273-82.

3. Cotton PB, Lehman G, Vennes J, et al. Endoscopic sphincterotomy complications and their management: An attempt at consensus. Gastrointest Endosc 1991;37:383-93.

4. Huibregtse K. Complications of endoscopic sphincterotomy and their prevention. N Engl J Med 1996;335:961-2.

5. Hofbauer B, Saluja AK, Lerch MM, et al. Intra-acinar cell activation of trypsinogen during cerulean-induced pancreatitis in rats. Am J Physiol 1998;275:G352-62.

6. Sherman S, Ruffolo TA, Hawes RH, et al. Complications of endoscopic sphincterotomy: A prospective series with emphasis on the increased risk associated with sphincter of Oddi dysfunction and nondilated bile ducts. Gastroenterology 1991;101:1068-75.

7. Wilson MS, Tweedle DEF, Martin DF. Common bile duct diameter and complications of endoscopic sphincterotomy. Br J Surg 1992;79:1346-7.

8. Chen YK, Foliente RL, Santoro MJ, et al. Endoscopic sphincterotomy-induced pancreatitis: Increased risk associated with nondilated bile ducts and sphincter of Oddi dysfunction. Am J Gastroenterol 1994;89:327-33.

9. Tarnasky P, Cunningham J, Cotton P, et al. Pancreatic sphincter hypertension increases the risk of post-ERCP pancreatitis. Endoscopy 1997;29:252-7.

10. Johnson GK, Geenen JE, Johason JF, et al. Evaluation of post-ERCP pancreatitis: Potential causes noted during controlled study of differing contrast media. Gastrointest Endosc 1997;46:217-22.

11. Freeman ML, Nelson DB, Sherman S, et al. Complications of endoscopic biliary sphincterotomy. N Engl J Med 1996;335:909-18.

12. Freeman ML, DiSario JA, Nelson DB, et al. Risk factors for post-ERCP pancreatitis: A prospective, multicenter study. Gastrointest Endosc 2001;54:425-34.

13. Vandervoort J, Soetikno RM, Tham TCK, et al. Risk factors for complications after performance of ERCP. Gastrointest Endosc 2002;56:652-6.

14. Friedland S, Soetikno RM, Vandervoort J, et al. Bedside scoring system to predict the risk of developing pancreatitis following ERCP. Endoscopy 2002;34:483-8.

15. Andriulli A, Clemente R, Solmi L, et al. Gabexate or somatostatin administration before ERCP in patients at high risk for post-ERCP pancreatitis: A multicenter, placebo-controlled, randomized clinical trial. Gastrointest Endosc 2002;56:48895.

16. Masci E, Toti G, Mariani A, et al. Complications of diagnostic and therapeutic ERCP: A prospective multicenter study. Am J Gastroenterol 2001;96:417-23.

17. Rabenstein T, Schneider HT, Bulling D, et al. Analysis of the risk factors associated with endoscopic sphincterotomy techniques: Preliminary results of a prospective study, with emphasis on the reduced risk of acute pancreatitis with lowdose anticoagulation treatment. Endoscopy 2000;32:10-9.

18. Loperfido S, Angelini G, Benedetti G, et al. Major early complications from diagnostic and therapeutic ERCP: A prospective multicenter study. Gastrointest Endosc 1998;48:1-10.

19. Mehta SN, Pavone E, Barkun JS, et al. Predictors of postERCP complications in patients with suspected choledocholithiasis. Endoscopy 1998;30:457-63.

20. Maldonado ME, Brady PG, Mamel JJ, et al. Incidence of pancreatitis in patients undergoing sphincter of Oddi manometry. Am J Gastroenterol 1999;94:387-90.

21. Sarner M, Cotton PB. Classification of pancreatitis. Gut 1984;25:756-9.

22. Sherman S, Blaut U, Watkins JL, et al. Does prophylactic administration of corticosteroid reduce the risk and severity of post-ERCP pancreatitis: A randomized, prospective, multicenter study. Gastrointest Endosc 2003;58:23-9.

23. Masci E, Mariani A, Curioni S, et al. Risk factors for pancreatitis following endoscopic retrograde cholangiopancreatography: A meta-analysis. Endoscopy 2003;35:830-4.

24. Aronson N, Flamm CR, Bohn RL, et al. Evidence-based assessment: patient, procedure, or operator factors associated with ERCP complications. Gastrointest Endosc 2002;56(Suppl):S294-302.

25. Concato J, Feinstein AR, Holford TR. The risk of determining risk with multivariate modes. Ann Intern Med 1993;118:201-10.

26. Fogel EL, Eversman D, Jamidar P, et al. Sphincter of Oddi dysfunction: Pancreatobiliary sphincterotomy with pancreatic stent placement has a lower rate of pancreatitis than biliary sphincterotomy alone. Endoscopy 2002;34:280-5.

27. Hogan WJ. Stenting the pancreas: Is this the solution to postERCP pancreatitis? Gastroenterology 1998;115:1591-4.

28. Rolny P, Anderberg B, Ishe I, et al. Pancreatitis after sphincter of Oddi manometry. Gut 1990;31:821-4.

29. Sherman S, Troiano FP, Hawes RH, et al. Sphincter of Oddi manometry: Decreased risk of clinical pancreatitis with use of a modified aspirating catheter. Gastrointest Endosc 1990;31:821-4.

30. Tarnasky PR, Palesch YY, Cunningham JT, et al. Pancreatic stenting prevents pancreatitis after biliary sphincterotomy in patients with sphincter of Oddi dysfunction. Gastroenterology 1998;115:1518-24.

31. Fazel A, Quadri A, Catalano MF, et al. Does a pancreatic duct stent prevent post-ERCP pancreatitis? A prospective randomized study. Gastrointest Endosc 2003;57:291-4.

32. Laugier R, Bernard JP, Berthezene P, et al. Changes in pancreatic exocrine secretion with age: Pancreatic exocrine secretion does decrease in the elderly. Digestion 1991;50:20211.

33. Freeman ML, Overby C, Qi D. Pancreatic stent insertion: Consequences of failure and results of a modified technique to maximize success. Gastrointest Endosc 2004;59:8-14.

34. Waldron RL, Luse SA, Wollowick HE, et al. Demonstration of a retrograde pancreatic pathway: Correlation of roentgenographic and electron microscopic studies. Am J Roentgenol Radium Ther Nucl Med 1971;111:695-9.

35. Roszler MH, Campbell WL. Post-ERCP pancreatitis: Association with urographic visualization during ERCP. Radiology 1985;157:595-8.

36. Sivak MV. Endoscopic management of bile duct stone. Am J Surg 1989;158:228-40.

37. Patel R, Tarnasky PR, Hennessy WS, et al. Does stenting after pancreatic sphincterotomy reduce post-ERCP 
pancreatitis in patients with prior biliary sphincterotomy?: Preliminary results of a prospective randomized trial [abstract]. Gastrointest Endosc 1999;49:AB80.

38. Elta GH, Barnett JL, Wille RT, et al. Pure cut electrocautery current for sphincterotomy causes less postprocedure pancreatitis than blended current. Gastrointest Endosc 1998;47:149-53.

39. Sherman S, Hawes R, Earle D, et al. Does leaving a main pancreatic stent in place reduce the incidence of biliary precut-induced pancreatitis? [abstract]. Gastrointest Endosc 1994;40:AB124.
40. Slot WB, Schoeman MN, Disario JA, et al. Needleknife sphincterotomy as a precut procedure: A retrospective evaluation of efficacy and complications. Endoscopy 1996:28:334-9.

41. Andriulli A, Leandro G, Niro G, et al. Pharmacologic treatment can prevent pancreatic injury after ERCP: A metaanalysis. Gastrointest Endosc 2000;51:1-7.

42. Murray B, Carter C, Imrie C, et al. Diclofenac reduces the incidence of acute pancreatitis after endoscopic retrograde cholangiopancreatography. Gastroenterology 2003;124:1786-91. 